\section{DE LA VIOLENCE À LA SUBJECTIVITÉ 1}

Lina María Vélez²

Psicoanalista

\section{Résumé}

A partir d'une thèse sur les incidences cliniques de la violence dans le discours et sur la subjectivité - menée auprès d'étudiants en Colombie - nous interrogeons, dans cet article théorico-clinique, les rapports entre lien social et symptôme comme constituants du fait humain. La violence interroge chercheurs et cliniciens, renvoyant à une multiplicité et une diversité de formes. En effet, si brute, si traumatisante que se présente la violence comme fait d'expérience, elle est indissociable d'une interprétation: laquelle met en jeu la dimension du sens, avec son corrélat de nonsens ; elle implique en même temps le sujet, avec ses déterminations et ses choix.

Une clinique de la violence ne gagnerait-elle pas à être éclairée par la psychanalyse ? Plus particulierement dans une attention portée à ce qui relève d'une position subjective et d'un rapport au savoir sur la violence de chacun?

\footnotetext{
1 El presente artículo es producto de la investigación realizada durante el Doctorado en Psicología clínica y psicopatología (Universidad Paris VIII) por la autora.

2 Doctora en psicología clínica y psicopatología (Universidad de Toulouse le Mirail Francia). Magister en Psicoanálisis (Universidad Paris VIII). Especialista en Psicología clínica (Universidad René Descartes Paris V). Psicóloga clínica: hospital de día para adolescentes, Ville d'Avray- Francia. Servicio de protección de la infancia, MelunFrancia. Docente del polo territorial de formación de educadores en la "Protección Judicial de la Juventud", Paris. Miembro de los Foros del Campo Lacaniano de Paris (Francia).
}

A partir d'une étude de cas, nous considérerons la position du sujet vis-à-vis du savoir et du lien social dans lequel il se trouve inscrit.

Mots-clés: violence, discours, Autre, clinique, psychanalyse.

\section{DE LA VIOLENCIA A LA SUBJETIVIDAD}

\section{Resumen}

A partir de una tesis sobre las incidencias clínicas de la violencia en el discurso y sobre la subjetividad, realizada con estudiantes en Colombia, cuestionamos, en este artículo teórico-clínico, las relaciones entre vínculo social y síntoma, como constituyentes del hecho humano. La violencia logra que los investigadores y médicos se cuestionen, remitiéndose a una multiplicidad y a una diversidad de formas. Efectivamente, la violencia, por más cruda y traumatizante que se presente, es inseparable de una interpretación; lo que involucra la dimensión del sentido con su correlativo de no-sentido, e implica, al mismo tiempo, al sujeto, con sus determinaciones y decisiones.

¿No se beneficiaría la clínica de la violencia de ser esclarecida por el psicoanálisis?, ¿y, especialmente, de prestar atención a aquello que tiene que ver con una posición subjetiva y al conocimiento sobre la violencia de cada uno?

A partir de un estudio de caso, consideraremos la posición del sujeto frente al saber y al vínculo social en el que se encuentra inscrito.

Palabras Clave: violencia, discurso, Otro, clínica, psicoanálisis. 


\section{FROM VIOLENCE TO SUBJECTIVITY}

\section{Summary}

In this theoretical-clinical article, based on a thesis about clinical incidence of violence in discourse and about subjectivity -carried out together with some students in Colombia, we wonder about the relations between social fabric and symptom as constituents of human element. The violence makes researchers and clinicians wonder about, referring to a multiplicity and diversity of forms. Indeed, even being a rough and traumatic experience, violence is inseparable of interpretation: which involves the meaning dimension and its correlate, the no-meaning one; it also involves the subject and its determinations and choices.

Wouldn't clinic of violence profit from being explained by psychoanalysis? Particularly, from focusing on what is part of a subjective stance and of knowledge on the individual violence?

From a case study, we will consider the stance of the subject towards the knowledge and the social fabric (s)he is in.

Key words: violence, discourse, Other, clinical, psychoanalysis.

Recibido: 02/04/09 Evaluado: 15/05/09 Aprobado: 19/05/09

\section{Du discours sur la violence}

Classiquement, trois composantes économiques, politiques et sociales sont décrites pour caractériser les causes et les diverses formes de violence en Colombie. Toutefois, les références théoriques des auteurs n'autorisent que des constructions partielles, selon la préférence accordée à l'une ou l'autre de ces approches.

Les différents travaux sur la violence nous placent face à une expérience historique où l'hétérogénéité du récit s'accompagne d'un morcellement fondamental du social et dans laquelle les révolutions et contre-révolutions sont dominées par l'illusion d'une rupture amenant une reconstruction. La violence est en fait prise dans de la continuité, elle commence par la volonté de maintenir ou de rétablir un ordre politique, elle se poursuit par un mélange de stratégies offensives et défensives, laissant un panorama inchangé, fait des mêmes structures sociales, de la même précarité de l'Etat. La violence affleure dans la mémoire individuelle et collective. Elle est inscrite dans le réel de l'histoire.

Les différentes descriptions concernant le lien social en Colombie nous montrent comment ces manifestations (guérilla, paramilitarisme) s'inscrivent en opposition à la domination de l'Etat, dans une protestation ambivalente (appel tout autant que refus) à l'encontre de l'Autre. Face à la montée en puissance de ces manifestations démonstrations, 
nous serions tentés d'élargir la perspective pour appréhender "ce qui fait symptôme » comme une forme de défection individuelle, en réponse à un malaise social.

Cette proposition vise à articuler le particulier et le collectif en déplaçant la dimension de la cause sur l'axe de l'Autre. ¿Comment allons-nous comprendre cette expression de malaise social?

Les recherches en sciences humaines et les travaux sur les problématiques mettant en avant le lien social, ont mis l'accent sur l'impact socialement désorganisateur des progrès technologiques, de la logique économique et de la civilisation moderne «individualisme, chute des idéaux, déclin de l'imago paternelle etc.».

Ces éclairages permettent de déplacer l'élaboration théorique du champ du pathos individuel aux caractéristiques du lien social et inversement. Ils laissent entendre l'idée d'une causalité linéaire dont toute symptomatologie serait en quelque sorte la traduction, l'expression directe d'un rapport ou d'une situation sociale ou économique.

Ce n'est pourtant pas l'une des orientations que nous avons retenues dans le cadre de notre recherche. II ne s'agit pas de faire l'économie des points de vues sociologiques, historiques et psychopathologiques, mais il nous semble nécessaire de mettre au travail d'autres sources théoriques, et particulièrement la catégorie du discours.

Penser le lien social d'une société en particulier, implique d'emblée de parler du discours, c'est-à-dire de la logique d'ordre du discours qui le détermine, de ce qui peut venir médiatiser les rapports du sujet à l'Autre social.

Tout discours peut être compris comme un appareillage déterminant des formes de lien social fondées sur le langage qui fait tenir les corps ensemble, qui doit permettre au sujet de trouver à s'y loger tout en parvenant à régler son propre rapport à la jouissance.

Le discours, en tant que dispositif de régulation de la jouissance, cherche à entraver cette dernière. II assure sa pérennité en engageant les sujets dans un procès économique leur permettant de récupérer des bribes de jouissance.

La jouissance introduite en creux par le signifiant, cette jouissance que le sujet perd à parler, va générer la ségrégation. "On » va se regrouper, on va se diviser, on va se déchirer autour des modes de jouissance, des modes de récupération de la jouissance compatibles avec son fantasme et le fantasme est par définition toujours singulier. (Sauret, 2000, p. 121) 


\section{Violence, transgression et jouissance}

Nous considérerons la violence dans son unité, malgré ses multiples manifestations. Ni la diversité des phénomènes de violence, ni les explications causales globalisantes ne peuvent empêcher que la violence soit en même temps «une». Elle témoigne d'une atteinte au lien social et suppose une position particulière du sujet qui s'y adonne.

La psychanalyse nous permet de progresser dans le déchiffrement de ce phénomène dans ses formes contemporaines: l'abordage structurel de la violence, dès qu'on y inclut la perspective de la subjectivité, offre la possibilité de contribuer à son élucidation.

Le sujet parlant est désirant, il est séparé de la jouissance, renouer avec elle implique une menace de disparition. Néanmoins le sujet désire cette jouissance dont il se soutient d'être séparé. Celui qui, sachant ce qui convient à la jouissance, la prélève directement sans le secours de la parole est appelé pervers. Même le névrosé qui a franchi les limites du langage reste fasciné par l'horreur.

Le sujet traite son rapport à la jouissance par les moyens du langage, il se sert particulièrement de la fonction paternelle. Du fait que le père est un signifiant, cette fonction paternelle permet à chacun de s'assurer de son appartenance à la communauté des humains en tant qu'homme ou femme.

La violence est proscrite pour ceux qui acceptent d'habiter la communauté des humains où règne la prohibition de l'inceste, du cannibalisme et de l'assassinat de son semblable. La loi définit les limites de la communauté, elle n'est que du langage, que du symbolique. Ainsi ce qui protège l'humain du non humain est constitué par du semblant. Les sujets disposent du système symbolique pour se défendre contre l'inhumain. Nous voyons que le surgissement de la violence est toujours possible, destructeur du lien social, et porté par les sujets eux-mêmes. Cette violence est celle du retour de la jouissance, dont le défaut demeure, au noyau de l'humain.

Le sujet est scindé structurellement à partir de la sujétion au langage, à la culture qui le détermine et l'institue dans son aliénation. II est ainsi constitué comme effet de l'ordre symbolique, caractérisé par son manque à être, par sa division qu'implique la méconnaissance de son désir et de sa jouissance. 
La violence est toujours présente, se déploie dans les rapports sociaux et exprime son intrication avec le lien social, la loi et sa transgression. C'est le paradoxe de la loi, qu'à partir de l'interdiction, elle ordonne le désir de la transgression et de l'excès. La violence est impliquée dans la subjectivité, compromet le désir, le corps et le lien social, mais s'installe dans l'arbitraire, hors de la régulation, et se trouve inscrite dans les rapports humains.

La violence se constitue comme une réponse spécifique face à une situation intolérable; ce qui signifie que chaque sujet, dans la même situation, peut réagir différemment; nous pouvons donc dire que toute réponse implique la responsabilité du sujet.

La violence convoque la dimension de l'acte et condense ses effets en laissant une trace. Dans cette perspective nous pouvons dire que la violence a un fondement structural qui noue trois éléments: le signifiant, l'acte et la trace ${ }^{3}$. Cette violence qui procède à la destruction du semblable peut se concevoir comme un passage à l'acte. Un acte qui exprime la transgression du corps et de la loi. Elle rend compte de la tendance à la destruction et à la mort du sujet envers lui-même et envers son semblable. L'anéantissement, la destruction et la mort de l'autre atteint le spectre de l'horreur.

Situons la violence et l'agressivité: à partir de l'idée que la violence se distingue par essence de la parole, nous la distinguons du terme d'agressivité. Cette différence s'établit au travers du principe suivant: seul peut être réprimé ce qui peut s'inscrire dans la structure de la parole, dans l'articulation signifiante. Ce qui correspond à l'agressivité et qui est donc symbolisé, peut être refoulé, interprété et analysé. C'est le champ de la rivalité, de l'assassinat du semblable, latent dans la relation imaginaire. La violence n'est pas symbolisée, elle est de l'ordre du réel et comme telle, impossible à intégrer dans l'ordre symbolique. Elle renvoie à la force du réel comme innommable.

La violence, l'agressivité, le passage à l'acte comme réponse du sujet, entraîne une conséquence: elle nous oblige à chercher sa source dans le sujet et à l'interroger sur ce qu'il fait de celle-ci, sur sa subjectivité. Elucider la violence dans le temps logique du sujet, demande de déchiffrer le moment de vacillement de ce dernier, l'instant du passage à l'acte dans la mesure où il reste fixé irrémédiablement à cet acte, marquant son histoire subjective.

II n'y a pas de violence sans sujet et avec lui est impliqué son corps. Ce qui nous oblige à transcender la notion d'organisme. C'est-à-dire que le corps s'instaure quand le réel

\footnotetext{
${ }^{3}$ La trace en tant que reconnaissance psychique de l'acte.
} 
de la chair et des organes est traversé par le langage et par l'image. A travers la dimension réelle, imaginaire et symbolique, le corps fait conjonction paradoxale de l'assemblage et de la fragmentation.

Ainsi, nous pouvons dire que le corps, dans sa tentative d'unicité, est une image propre de la dimension imaginaire, il est traversé par un nom dans la dimension du signifiant, c'est le lieu du pulsionnel et le champ privilégié de la jouissance.

La violence s'inscrit dans le corps, dans ses différentes dimensions. Dans la dimension imaginaire en tant que corps qui s'exalte, et dans la dimension symbolique comme corps qui se fracture ou se désintègre. Le corps en tant que réel souffre et jouit.

La violence apparaît comme une rupture qui ouvre à l'excès, qui se réjouit, qui se délecte dans un corps; l'émergence de la jouissance dans l'acte destructeur où l'autre acquiert le statut d'objet dégradé, objet découpé, objet mort, se situe au-delà du prohibé, du fondement de l'ordre social qui interdit de tuer son semblable.

Nous pouvons dire que, dans les dimensions et logiques de la cruauté excessive, celle que Lacan a nommée «sévice» (Lacan, 1988, p. 345), s'exerce une humiliation du sujet dans son existence, le réduisant à un objet, le dépouillant, le destituant de sa dignité dans un mouvement d'annulation subjective qui compromet son existence comme être capable d'exprimer un désir. L'avilissement, qui réduit à rien son existence désirante, l'abolit en tant que sujet.

Prendre position face à la violence demande de déchiffrer la jouissance qui l'habite. Ces éléments théoriques nous permettent de dire que la violence à laquelle font référence les sciences sociales, cette violence inédite qui fait irruption est un aspect, une particularité du lien social en Colombie, qui compromet le sujet dans sa jouissance; et comme telle, elle échappe à l'ordre symbolique, parce que ce dernier n'arrive pas à opérer son ordonnancement, et sa régulation.

Cette violence est une mise en scène qui décline l'Autre ${ }^{4}$ : d'une part ordre symbolique particularisé pour chaque sujet qui semble avoir sa face pacifiante; mais aussi face mortifère, en tant qu'introductrice des mandats qui incitent à l'excès et justifient l'effacement du semblable.

\footnotetext{
${ }^{4}$ L'Autre désignant l'Etat à travers ses institutions selon le cas clinique ci-après. 
La violence est devenue un moyen légitime, mais non légal, de résoudre divergences et conflits à travers un échange dans le réel, exacerbé de transgressions, de destruction, d'excès, d'actes de profanation et de barbarie impliquant le corps, le versement du sang et la mort. Ainsi "la légitimité de la violence ne tient en effet qu'à son rapport au discours, au lien social et à l'acte." (Sauret, 2000, p. 143). La violence a trouvé son support en se justifiant de l'idéal: "se défendre de l'autre".

Nous considérons que cette violence, en tant qu'acte, est nouée au signifiant et peut disposer, sans même que le sujet le sache, de choix décisifs pour son destin. En se configurant un devenir par la voie du signifiant et en se reproduisant de façon énigmatique, la violence en Colombie profile l'organisation des modalités de jouissance et transcende les générations, en raison de son inscription dans le symbolique, c'est à dire dans ce qui peut se collectiviser et se léguer à la filiation. Malgré son inscription symbolique, la violence opère comme un savoir essentiellement ignoré par le sujet. Un acte de violence inséré comme signifiant, acquiert son pouvoir et laisse une trace ineffaçable. Nous pouvons dire que son pouvoir de commandement et son effet de portée inaugurale, donne lieu à une constitution subjective.

Dans ce contexte, la violence référée à la force maintient l'ordonnancement de l'humain, le pouvoir régulateur de la loi et des pactes et de la même façon, la transgression de la loi implique avant tout la transgression du lien social, du sexuel, du corps. Nous considérerons la violence comme un symptôme particulier bien que non spécifique du lien social. Nous situerons la violence au cas par cas, comme témoignage d'une position subjective qui s'inscrit dans un ordre du discours.

Parler de position subjective, c'est considérer la manière dont le sujet «\$ $\$$ prend acte de l'aliénation signifiante qui préside à son effectuation (niveau primaire de la castration), mais aussi comment il vient s'inscrire dans le social en tant que sujet du désir (niveau secondaire de la castration) référencé sur un mode contingent à la loi phallique. 


\section{Etude de cas}

Dans le cadre de notre recherche, nous avons rencontré une vingtaine d'adultes entre 25 et 45 ans, des étudiants qui voulaient parler de la violence en Colombie. Deux d'entre eux avaient fait partie de la guérilla ${ }^{5}$ et d'un groupe paramilitaire.

Dans les entretiens de recherche nous ne pouvons pas parler de l'existence du transfert tel qu'il surgit dans le cadre d'une analyse, néanmoins chaque entretien propose une place particulière à partir de laquelle les participants à notre recherche acceptent de parler.

Le sujet entrait dans un dispositif de parole, il rendait compte de son histoire, de sa situation actuelle à l'égard du lien social, témoignait de son ressenti, de ses attentes et de ses projets.

"Je suis inscrit dans cette institution. Néanmoins je sens que cet endroit ne me correspond pas. Depuis l'année dernière je suis inscrit en BTS d'agronomie, je crois que je vais laisser les études, je ne pourrai pas tenir ici". G. est un homme de 27 ans qui avait fait partie de la guérilla pendant 10 ans. Il a eu un long parcours dans des groupes armés illégaux. Il est témoin des difficultés de la mise en place des politiques de réintégration à la vie civile concernant les jeunes qui quittent les groupes aux marges de la loi. C'est à partir de ce discours sur la violence, de sa «réintégration à la société» à une place d'étudiant qu'il ne peut pas assumer, qu'il construit sa propre histoire. Tout en s'identifiant comme spectateur et protagoniste de l'évolution du conflit armé auquel il a participé.

\section{Le récit familial et l'exclusion.}

G. est le second d'une fratrie de deux enfants.

"Mes parents ont vécu un moment difficile parce qu'ils avaient beaucoup de dettes, l'argent que gagne mon père ne suffisait pas. Pour résoudre cette situation on a dû aller vivre ailleurs. Donc on va vivre chez ma grand-mère. Et c'est quelque chose de difficile pour mes parents. Pour mon père notamment...il faut placer les choses dans notre contexte culturel où....pour un père de famille, être dépendant de sa belle-mère c'est une sorte de dégradation. Ça a été quelque chose d'assez difficile à vivre pour mon père. Tout d'un coup on n'était plus chez

\footnotetext{
5 "El surgimiento de las guerrillas en los anos 60 se produce de manera simultanea en referencia al mundo urbano y al mundo rural. Dicho surgimiento es impulsado, por una parte por el radicalismo de los actores urbanos, salidos muchas veces de las clases medias, que han llamado a la destrucción de un régimen que les parece entrabar el advenimiento de la modernidad en todos los campos, sociales, políticos, culturales; y por otra, por la persistencia de la violencia en las zonas rurales y la disponibilidad relativa de una parte de la población campesina para vincularse a una movilización armada". (Pécaut, 2003, p. 46)
} 
nous, nous étions chez la grand- mère. Alors que c'est lui le père enfin, ainsi c'est, c'est la grand-mère qui est devenue la responsable de la famille."

Dans la famille la situation est vécue comme une dégradation de la figure du père, dont les effets traversent toute la configuration des relations. G. décrit un père dont l'absence est le résultat d'une situation sociale. Pour lui, si le père n'arrive pas à subvenir aux besoins de la famille, c'est parce qu'il y a un contexte social qui l'en empêche. "Mon père travaillait beaucoup, mais il gagnait très peu, de toute façon il était toujours exploité par l'entreprise de l'Etat qui n'a jamais voulu lui donner un contrat de travail stable". En conséquence, c'est l'Autre du social, incarné par les entreprises du gouvernement dans la société colombienne, qui se présente pour G. comme un agent de ségrégation.

\section{La violence comme « son autre face»}

G. fait référence à sa propre violence, à ce qu'il nomme dans son témoignage son «autre face». Au travers de celle-ci, il décrit une série de manifestations de la pulsion dont il essaie de nier le caractère violent. "Enfin je crois que je ne suis pas quelqu'un de violent... je (ne) suis pas violent ni rien de tout ça. Mais je suis quelqu'un d'autre quand je deviens violent". Malgré son refus de reconnaître sa propre violence, il s'est trouvé confronté à celle-ci. Souvent ce sont des effets imaginaires, en miroir, qui le conduisent à rencontrer ces manifestations indésirables de la pulsion. "Je jouais avec mes camarades du lycée, il fallait montrer qui était le plus fort, tout commençait bien, mais ensuite j'avais envie d'achever mes copains, je ne pouvais pas maîtriser ma force, et bon... j'étais pris dans une force extraordinaire, je ne m'arrêtais pas". Nous pouvons dire qu'il se perd dans un rapport imaginaire où il n'y a pas de limites entre lui et son semblable. C'est comme s'il essayait de récupérer une part de la jouissance perdue en retournant à un état d'indifférenciation dans lequel il ne se reconnaît pas, et où les limites auraient tendance à disparaître.

D'autre part, il décrit une occasion dans laquelle il avait décidé d'interpeller son père en lui reprochant son incapacité à résoudre les difficultés économiques de la famille. Face à la non-réponse de celui-ci, G. réagit en le frappant.

"J'étais particulièrement contrarié. Je me suis énervé et je lui ai renvoyé tout ça à la figure. Comme il ne répondait pas, je me suis énervé un peu plus et je lui ai tapé dessus. Je n'aurais jamais dû faire ça, jamais. Encore aujourd'hui, quand j'y repense... bon. Mais bon, voilà c'est fait, c'est fait. Je ne peux pas revenir en arrière".

Cette situation de détresse du père, semble être perçue comme une défaillance, il réclame à ce dernier d'occuper sa place. 
Ce qu'il appelle son «autre face» semble être la manifestation de sa propre violence ainsi que le dépassement de l'angoisse qui l'inonde. G. fait toujours le même rêve, qui semble témoigner de la lourdeur d'un malheur menaçant. "Je rêve qu'une catastrophe peut arriver à mes proches et notamment à ma mère. Je vois que tout est détruit autour de moi, mais ce sont les murs de la maison qui s'effondrent sur nous, plutôt sur ma mère et moi".

\section{Le rapport à l'autre}

Face à la mort le sujet ne se défausse pas, mais reste un peu perplexe. C'est un point de savoir au sens où tout le monde "mourra " un jour. L'impossible à savoir renvoie le sujet à un fait d'incroyance: "c'est une fois qu'on est mort. C'est fini...C'est fini je crois". De même, pour le paradis, il n'y croit pas: "je crois si je le vois venir...". La mort ne renvoie qu'à la vue de la mort, c'est-à-dire que c'est du réel que le sujet « voit » venir ce qui le concerne: "si je le vois, j'y crois. Si je ne le vois pas, je n'y crois pas". Ces énoncés concernant la mort sont surprenants parce qu'il a été souvent confronté à celle-ci. Cependant il va nous parler de la mort dans le deuxième entretien d'une façon différente:

"quand j'étais dans la " guérilla " la mort était quelque chose que je voyais souvent. On devait faire justice dans le quartier, on chassait les voleurs, les dealers. Mais s'ils refusaient de quitter le quartier, il fallait les tuer. C'était pour nettoyer le quartier, on devait garder un certain ordre et faire respecter les habitants... II y a des gens qui doivent mourir parce qu'ils font beaucoup des dégâts dans la société."

G. nous montre qu'il se positionnait en tant que justicier, c'est à dire qu'il s'autorisait d'une façon tyrannique à déterminer qui pouvait vivre ou pas. A partir d'une pratique ségrégative qui n'était pas très différente des stratégies d'exclusion qu'il critiquait de la part de l'Etat, $G$. justifiait ses actes. Nous voyons comme «l'autre» dans ce cas, n'était pas considéré comme un semblable, il était réduit à un objet, un déchet nuisible à la société et qu'il fallait détruire. II nous a dit que la mort c'est quelque chose qu'il voyait souvent, peut être était-ce une façon de dire qu'il ne pouvait pas envisager la sienne propre. Pendant les entretiens nous avons constaté que face à l'acte de tuer il parlait toujours à la troisième personne du pluriel. Cependant il n'a pas évoqué l'acte meurtrier d'une façon directe.

\section{Le rapport à l'Autre et le lien social}

G. parle de son histoire faisant une analyse de sa participation et de sa place dans la "guérilla». Face aux dynamiques sociales il se montre comme un protagoniste à partir du moment où il intègre le groupe. Dans un premier temps il essaie d'établir un lien entre le désarroi social et son insertion dans la «guérilla». Dans un deuxième temps, il doit se retirer du groupe parce que l'Etat, au travers d'un «Programme de Réinsertion », propose aux 
jeunes de retourner à la vie civile. II reprend les études et commence une formation sans pouvoir répondre aux demandes ni à cette figure de l'Autre.

D’abord, G. reconnaît que son désinvestissement scolaire actuel n'est pas nouveau. II a déjà été confronté à cette situation. Les difficultés concernant son rapport au savoir ont toujours été présentes. Néanmoins c'est à la suite d'une agression quand il avait 16 ans, qu'il a quitté définitivement le lycée. Lorsqu'il se promenait avec un copain hors de son quartier il est victime de jeunes qui lui volent sa montre sa veste et le « cachent » dans une cave en le menaçant avec des armes pendant deux jours. Cette expérience constitue une véritable intrusion du réel: "je me suis fait agresser par cinq personnes, je pense que c'est beaucoup à la fois, j'ai été choqué sur le coup, puis, après, j'ai essayé de m'en remettre un peu, mais... quoi, je ne sais pas si j'ai réussi. "L'impact traumatique se poursuit "j'étais à bout de nerfs quand je suis sorti, ils m'ont laissé partir. J'avais... j'avais tout cassé quand je suis rentré chez mes parents, et après j'ai eu peur de ressortir". La peur de l'autre, des autres, est désormais au rendez vous et l'espace social devient lui aussi menaçant. II reste chez lui pendant quelque temps malgré les reproches de ses parents et les appels du lycée pour qu'il y retourne. "Je ne sais pas pourquoi mes copains disaient que probablement j'avais fait quelque chose, que cette agression n'était pas gratuite, ils se sont tous mis à s'écarter de moi parce que j'étais devenu très agressif, je voulais me battre avec n'importe qui". De cette mésaventure comme de la mise à mal de ses ressources subjectives, l'Autre ne veut rien savoir. Cette incompréhension est vécue comme une violence, une injustice exercée à son endroit. "Je n’ai pas cherché à parler avec eux, je me suis barré du lycée".

Lorsque le sujet se sent repoussé ou rejeté, il privilégie l'autodéfense à la soumission "ça ne va pas rester comme ça". Si G. laisse tomber sa scolarité, ce n'est en aucun cas pour recevoir une sanction de l'Autre, ni pour entrer en résistance. Cette sortie est plutôt du registre du passage à l'acte quand, en claquant la porte, le sujet traverse une limite "ça a été fini". II s'en trouve mortifié: "pendant ce temps, je n'ai rien fait, je suis resté chez moi". C'est une solution extrême qui, dans l'économie psychique, nécessite des échappatoires. Car chez lui, G. tourne en rond, étouffé, s'agite, cherche à comprendre ce qui, dans tout cela, doit certainement le concerner, en passe par les maux du corps.

Le fait d'éviter la rencontre avec l'Autre du social renvoie à une impasse subjective que le sujet ne cesse de rencontrer. II pense qu'il était "malade des nerfs », il ne sait pas comment faire face à cet afflux de sensations et de tensions dans son corps: "Je m'énervais tout seul". 
Cette situation énerve aussi son père. II est donc poussé à s'en aller, d'après son père il devait apprendre à se défendre. Pris en charge, raccordé en quelque sorte à son moi auxiliaire (parrain, ami), G. va vivre dans un autre quartier chez un oncle où il va rencontrer un groupe de copains qui vont l'accueillir. L'espace s'agrandit, le mouvement permet de limiter cette tendance à l'agitation désordonnée, fixe un cadre, un contenant. II semble trouver une place dans ce groupe de jeunes qui se caractérise en faisant la loi dans le quartier. C'est-à-dire qu'ils s'autorisent d'eux-mêmes à chasser les voleurs, les prostituées et les dealers. L'introduction de G. dans la « guérilla » va se produire à ce moment là.

\begin{abstract}
"Dès que j'ai rencontré ces gars j'ai eu la sensation de commencer à me tranquilliser, là... j'étais protégé, j'étais pris en charge par des gens qui pouvaient me défendre de l'agression que j'avais subie, des dangers et des menaces que j'avais commencés à ressentir depuis ce jour. C'est comme si j'avais eu la possibilité de canaliser la violence que je ressentais. Et puis finalement c'était pour une bonne cause. Nous défendions les commerçants du quartier, les femmes et les enfants contre les délinquants."
\end{abstract}

Pourtant, nous ne pouvons discerner ici aucun calcul, aucun jeu désirant. Plutôt la soumission à quelque volonté obscure ou banale de l'Autre. Nous pouvons dire à titre d'hypothèse qu'il s'agit d'un appareillage minimal qui a comme fonction de faire face à l'angoisse ressentie. Et qui n'est qu'une réponse dérisoire face au choix forcé et précoce du sujet.

G. semble banaliser le fait d'avoir intégré des groupes illégaux à partir de 16 ans. II ne peut pas, pour l'instant, établir un lien entre les conséquences de l'agression et sa façon de s'introduire et de participer à ce groupe. Pour G., l'ambiance du quartier suffit comme facteur déclencheur: "Les jeunes des quartiers défavorisés appartiennent toujours à des groupes, des bandes, je crois que c'est une façon d'être ensemble, de nous reconnaître et de nous défendre. Le contexte social fait que ces groupes existent, on n'appartiendrait pas à ces groupes s'ils n'essayaient pas de prendre, de récolter les jeunes". Pour expliquer son entrée dans le groupe armé il fait une description du contexte social dans lequel il a grandi et qui prétend justifier son choix. A travers une explication logique entre l'offre et la demande, le choix et l'engagement se font effectifs comme s'il s'agissait d'établir une relation entre la précarité sociale et le marché d'objets disponibles.

"Je crois que.... chacun a son histoire mais, il y a quand même des choses qui se recoupent, c'est-à-dire que c'est singulier pour chacun, mais dans le milieu social, les jeunes ont à peu près la même histoire que moi. Les groupes minoritaires en tout cas, on va dire ça comme ça... quand ils se retrouvent, quand ils se retrouvent dans ces groupes, il y a quand même des questions d'identité qui sont en jeu, je pense...en tout cas pour moi c'était le cas."

Selon lui, le groupe armé, la « guérilla » en tant que pratique ségrégative, devient un espace d'union entre les minorités. Le modèle d'identification est pour lui le fait de lutter contre le 
chaos qui règne dans le quartier et de faire la loi dans la cité. Essayer de se positionner comme celui ou ceux qui font la loi est une forme d'incitation à la révolte contre une société inégalitaire et excluante. Se substituer aux institutions qui représentent l'Etat est une façon de mettre en évidence l'inefficience de celui-ci. D'où, le groupe qui sert d'outil de révolte contre un Etat défaillant face auquel il faut se regrouper pour s'auto-défendre.

Le fait d'appartenir, de s'engager dans ce groupe, constitue pour G. une sorte d'intériorisation des effets de la ségrégation. Nous pouvons dire à titre d'hypothèse qu'il s'agit dans ce cas d'une position masochiste par laquelle le sujet cherche à faire appel au désir de l'Autre tout en se faisant lui-même déchet.

"Je peux dire que nous, enfin moi à l'époque, quand je me suis engagé dans ce groupe, j'ai retourné la violence contre moi-même, la violence qui était déjà là en moi et c'était aussi forcément la violence de la cité où là, la violence, la violence qu'on pouvait vivre, qu'on pouvait vivre...à l'intérieur dans nos relations c'était extrêmement violent. C'était une violence terrible. C'était sombre, c'était de l'isolation, avec tout ce que ça peut entraîner de violent à vivre. On décide de s'engager dans les groupes armés sans le savoir, ce n'est pas une décision, enfin, c'est vouloir vivre la guerre. Guerre contre soi, contre les autres. II est nécessaire de faire très attention à ne pas aller dans les autres quartiers. Chaque zone a des groupes constitués pour gérer les problèmes du quartier. II faut s'informer tout le temps des gens qui arrivent. Des fois la police essayait de rentrer au quartier. Chasser les dealers c'est le plus compliqué, ils subornent tout le monde. C'est vraiment infernal, Je crois que l'on peut assimiler ça à une situation de guerre. Dans mon cas ça a durée 10 ans et ça devenait un mode de vie".

G. sent qu'il s'était engagé dans ce groupe sans savoir comment, pourquoi, ni à quel moment il y avait pris racine. Dans son récit il y a une sorte d'analogie entre les groupes armés et le fait de vivre en guerre. G. déplie son analyse à partir de deux éléments omniprésents dans l'engagement. D'abord, faire du groupe armé une modalité de vie, et vivre dans la guerre. Ceci conduit le sujet à un état d'urgence où il semble impossible d'arrêter la précipitation des événements. Le sujet se trouve sans aucune capacité d'avoir les conditions minimales de reprendre une autre option de vie. Cependant il existe dans le monde des «groupes armés, comme dans la guerre, des séquelles.

Cette expérience montre bien que le vrai problème ne se trouve pas dans le groupe lui-même, ni dans l'ambiance qui l'entoure mais, dans un ensemble de significations qu'il porte avec lui. Ce sont peut être les effets de l'inconscient, et de sa propre violence qui le conduisent toujours à se retrouver dans ces groupes. Nous pouvons dire que G., tout au long de son récit, fait référence à une figure conformée par l'Etat et la cité. Il dénonce un Etat qui n'accomplit pas sa fonction, qui comme son père n'est pas garant de la loi ni de l'ordre. La fonction paternelle n'a pas réussi à limiter la jouissance de G. qui se traduisait par de la violence. L'Etat n'arrive pas à s'imposer ni à faire barrage à la jouissance dans le quartier. II 
ne représente pas le phallus qui devrait garantir la loi à l'intérieur de la cité. Les quartiers sensibles où G. a habité, sont des lieux où les jeunes gens se perdent dans la jouissance de la violence. Ainsi, le récit concernant la cité nous montre d'une part qu'il condamne l'Etat comme impuissant et négligent face à la condition des jeunes, ne pouvant rien faire pour éviter leur chute dans le désespoir social. D'autre part, son entrée et sa participation dans le groupe armé» est peut être une stratégie qui lui permet de trouver un cadre pour exercer, légitimer sa violence face aux autres, s'autoriser à faire la loi et trouver une place dans le social.

\section{Que faire de la violence, du discours sur la réinsertion?}

Dans son récit, G. explique que dans ce lieu de ségrégation que sont les groupes de la "guérilla», il y a ceux qui arrivent, comme il l'a fait, pour "chercher une solution à leurs problèmes, à un mal qu'ils ne peuvent pas supporter". Il semble reconnaître dans la figure du sujet qui fait partie de la «guérilla» quelqu'un qui s'est trouvé une solution pour résoudre sa détresse sociale.

Puis il doit les quitter:

"Avec le temps le groupe commençait à s'agrandir et à avoir beaucoup de pouvoir au-delà du quartier. Même si nous exercions la violence il y avait une certaine tranquillité dans le quartier, c'était grâce à nous que cela était possible. Le problème a surgi quand l'argent "sale " a commencé à pénétrer dans le groupe. C'est-à-dire qu'à un moment donné il fallait s'organiser mieux pour pouvoir se défendre. On avait commencé à demander de l'argent aux commerçants pour pouvoir continuer à fonctionner. Finalement on s'est laissé séduire par l'argent qui venait des trafiquants de drogues. Il y avait une rivalité à l'intérieur du groupe, chacun voulait avoir des objets, des armes, des voitures, des choses... II fallait donner des fonctions à chacun. C'est de cette façon que le groupe a commencé à se détruire et que la situation est devenue chaotique. La police s'est mêlée de cette situation parce que le groupe avait obtenu un pouvoir économique, c'était la seule chose qui les intéressait. On existait depuis quelques années, jamais l'Etat n'était présent. On ne les avait jamais intéressé, la vie dans des quartiers défavorisés non plus... débrouillez-vous, entretuez-vous, on n'y peut rien."

La militance dans la « guérilla » est une façon pour G. de faire avec la violence.

Cependant son choix de faire partie de la « guérilla » reste pour nous énigmatique et nous questionne. Peut-être s'agit-il d'une alternative pour se faire une place dans la société ? II jouit du discours concernant la détresse sociale dans toutes ses formes. II montre comment les jeunes sont pris dans la logique de consommation des objets proposés par le marché. II situe la problématique de l'appartenance aux groupes des «guérilleros» comme une difficulté qui a une racine sociale plus qu'individuelle. Bien que son discours puisse paraître élaboré, il soutient la logique de ségrégation qu'il dénonce. Son explication semble le débarrasser de sa propre responsabilité dans sa condition. G. a été blessé dans un des 
affrontements entre la " guérilla » et l'Armée, il a été soigné et ensuite arrêté par la police. II dit avoir été obligé de faire partie d'un programme de " Justice et paix » que propose l'Etat aux jeunes qui veulent retourner à la vie civile. A ce moment là il s'agissait d'aller en prison ou d'intégrer ce programme. "Je ne pouvais aller en prison, ce serait comme être mort en vie. Là-bas je ne pourrai pas me défendre, le comble de la violence c'est la prison". Pour G., retourner à la vie civile c'est une injonction qui vient de l'Autre social. ¿Qu'est ce que la vie civile pour lui?

"J'ai dû me soumettre à un suivi de la part de la justice depuis trois ans, ils me surveillent, j'ai eu mon bac, je ne peux pas revenir en arrière. Mais je sens que tout ça n'a pas de sens. J'ai peur, j'ai peur de m'effondrer. Quand je suis dans la rue je me méfie, cela m'arrive depuis que j'ai laissé le groupe. Je ne sais pas ce qui peut m'arriver par derrière. Je ne trouve pas ma place dans la formation que j'essaie de faire à l'institut d'agronomie. Ce n'est pas mon monde, je n'y appartiens pas, je vais finir mal, tout ça ce n'est qu'une comédie. Je crois que ce sont les médicaments qui me font tenir. Les institutions ne comprennent rien à ma situation, ni à ma détresse"

Même si G. dit «être obligé » de suivre ce programme, il n'a pas cherché à se débarrasser des contraintes imposées par l'Autre. II n'a pas pris la fuite pour l'instant. Le cadre lui a permis quelque temps de limiter et contenir sa violence. A un moment donné, cette interaction en apparence stable qui le soutenait, ne fonctionne plus. Les problèmes de G. conduisent l'Autre de la loi à intervenir. Le juge est appelé à prendre le relais, à s'occuper de lui. G. s'est retrouvé en possession d'armes à l'intérieur de l'institut où il essaie de faire une formation. II a ainsi réussi à drainer tout un cortège des petits autres (assistante sociale, éducateurs) à sa suite.

\section{Symptôme et lien social}

Nous pouvons dire que c'est le rapport à la loi qui constitue le coeur de la problématique de G. II y a une défaillance symbolique qu'il essaie de réparer. II ne s'agit pas de se réinsérer dans la société et de faire semblant de s'intéresser au savoir. II s'inscrivait dans le lien social en faisant partie du groupe. Le recours à la violence vient répondre à une intrusion massive de jouissance et permet au sujet d'accommoder une angoisse vertigineuse, placée aux bords d'un trou où ce ne serait pas l'approche de l'objet du désir mais un défaut radical d'être, qui serait en jeu. A partir des ces trois entretiens, il ne nous semble pas pertinent de parler en termes de structure, mais il est important de montrer la place qu'occupe le recours à la violence dans son économie subjective. La peur cherche son objet dans une méfiance projective vis-à-vis de l'autre comme vis-à-vis de l'environnement. G. ne s'en cache pas. Dans la rue tout seul, il est désormais inquiet. Seule la présence de ses amis du groupe, enrôlés dans une fonction de sécurité, était pour lui rassurante. Dans un tel contexte, tout 
espace social différent de la «guérilla» peut être un lieu de confrontation à l'autre potentiellement persécuteur.

\section{Conclusion}

Nous avons choisi de présenter ce cas dans une tentative d'éclaircir les particularités du lien social et de la violence en Colombie. A partir d'une méthodologie référencée à la psychanalyse, l'étude clinique détaillée met en évidence la particularité de la position subjective dans les accidents ou ruptures du lien social.

Dans le cas présenté, la violence et le rapport du sujet à celle-ci, tiennent à une conjoncture où la question de l'être du sujet est en jeu. Le recours généralisé à la violence est une particularité de ce lien social. Mais quel que soit son mode, elle peut être reconnue comme un fait de structure, c'est-à-dire qu'elle renvoie, par delà l'expressivité du phénomène, à une prise de position subjective qui peut éclairer rétrospectivement la structure. Ce qui a pour conséquence de mettre en question, malgré la mise en évidence des facteurs convergents, tout modèle de causalité; comme de pointer le caractère vain de toute approche cumulative des déterminants socioculturels, familiaux, etc.

Même s'il s'agit d'entretiens de recherche, il y a des mouvements transférentiels plus ou moins massifs. ¿Qu'en est-il pour le psychologue clinicien dont la pratique se résume à trois entretiens? G. semble s'engager dans un dispositif de parole qu'il sait limité. La prise en compte des éléments transférentiels dans la recherche nous met face à ces deux questions: ¿que vient-il nous adresser et de quelle place l'entendre?

Au-delà d'un désir d'être entendu, s'agit-il pour G. de se raconter comme pour répondre à une injonction présupposée, où utilise-t-il ce dispositif qui lui est proposé pour mettre en place un questionnement sur lui-même ?

D'autre part, son récit met en cause du point de vue clinique la pertinence des programmes de réinsertion qui répondent à une logique sociale, mais ne tiennent pas compte de la dimension subjective de ceux qui s'engagent dans ces groupes armés ilegaux. En nous écartant de toute référence au modèle «biopsychosocial » dominant, qui ne parvient à faire argument qu'en barrant ou déniant la prévalence de la dimension subjective, nous mettons en cause le privilège accordé aux inégalités économiques et sociales pour expliquer le malaise social. 
La violence est une réponse palliative du sujet face à l'émergence d'un réel insupportable ou inassimilable, notamment lorsqu'elle entraîne une rupture du lien social, procède d'un " choix forcé » qui dépasse les limites du champ de l'Autre social. Certes, la position du sujet ne se soutient que d'un rapport au savoir et de l'adoption d'un mode particulier de traitement de la jouissance. Mais, comme notre étude de cas nous l'indique, le rapport à la violence permet aussi d'éclairer comment le sujet échoue à s'inscrire dans le lien social, et de quelle façon il avait jusque là tenté ou réussi à y placer sa singularité.

La violence dépend de l'existence d'un discours qui la reconnaît tout en la dénonçant comme telle, parce qu'elle le dépasse. Ainsi peut-on dire que la violence apparaît comme un accident du lien social.

Par rapport au discours comme lien social, la violence s'impose comme limite: limite inhérente au discours et limite rencontrée par le discours. Elle peut intervenir dans plusieurs cas et à plusieurs niveaux: quand le lien social dans son ensemble se dégrade ou quand l'une de ses formes tend à prédominer sur les autres et à s'imposer par la force; comme protestation, révolte, attaque ou critique.

La violence ne serait pas seulement inscriptible comme un objet de savoir mais permettrait de révéler dans son après-coup la dialectique interne, l'économie de jouissance du discours qui constitue la base des procédures techniques trouvant à s'exercer au sein du système politique.

\section{Bibliographie}

Lacan, J. (1988), "Les formations de l'inconscient", Le séminaire, livre V. Paris: Seuil. (1991) "L'envers de la Psychanalyse", Le séminaire XVII, Paris : Seuil.

Pécaut, D. (2003) "Violencia y política en Colombia" elementos de reflexión. Medellín: Ed. Hombre nuevo y Universidad del Valle.

Sauret, M-J. (2000) "Psychanalyse et politique" Huit questions de la psychanalyse au politique; Toulouse: collection Psychanalyse P.U.M.

Vélez, L. (2007) Le lien social et le symptôme: Incidences cliniques de la violence dans le discours et sur la subjectivité d'étudiants universitaires en Colombie. Thèse pour le doctorat en psychologie clinique et psychopathologie. Université de Toulouse le Mirail, Décembre. 